\title{
Abdominal Cocoon or Encapsulating Peritoneal Sclerosis: A Rare Cause of Small Bowel Obstruction
}

\author{
Omar Lasheen ${ }^{1}$, Mohamed ElKorety ${ }^{2}$ \\ ${ }^{1}$ General Surgery Department, Royal Oldham Hospital, Pennine Acute Trust, Manchester, UK \\ ${ }^{2}$ General Surgery Department, West Hertfordshire Hospitals NHS Trust, Hertfordshire, UK
}

\section{Doi: 10.12890/2020_001972 - European Journal of Case Reports in Internal Medicine - @ EFIM 2020}

Received: 23/08/2020

Accepted: $11 / 09 / 2020$

Published: $12 / 10 / 2020$

How to cite this article: Lasheen O, EIKorety M. Abdominal cocoon or encapsulating peritoneal sclerosis: a rare causa of small bowel obstruction. EJCRIM 2020;7: doi:10.12890/2020_001972.

Conflicts of Interests: The Authors declare that there are no competing interests.

This article is licensed under a Commons Attribution Non-Commercial 4.0 License

\section{ABSTRACT}

Encapsulating peritoneal sclerosis (EPS), also known as abdominal cocoon syndrome (AC) or sclerosing encapsulating peritonitis (SEP), is an uncommon condition typically presenting with features of bowel obstruction. We present the case of a 41-year-old male patient who presented to the accident and emergency department with a 7-day history of abdominal pain. Contrast CT of the abdomen and pelvis was ordered and was suggestive of small bowel obstruction involving most of the small bowel with no apparent transition point. Laparotomy showed a tough whitish fibrous membrane encasing the entire length of the small bowel. Advances in CT have made diagnosis possible before a decision on surgical intervention is made.

\section{LEARNING POINTS}

- Despite being a rare cause of bowel obstruction, based on the clinical presentation and CT findings, abdominal cocoon syndrome should be included in the differential diagnosis.

- CT of the abdomen is the investigation of choice for most cases of bowel obstruction and can be very helpful in reaching a diagnosis before operative management is undertaken.

- Laparotomy is the usual choice for management, but laparoscopy can be considered either to establish the diagnosis or to deal with the abdominal cocoon based on the surgeon's clinical judgement and experience.

\section{KEYWORDS}

Encapsulating peritoneal sclerosis, sclerosing encapsulating peritonitis, bowel obstruction, intestinal obstruction, abdominal cocoon syndrome

\section{INTRODUCTION}

Encapsulating peritoneal sclerosis (EPS), also known as abdominal cocoon syndrome (AC) or sclerosing encapsulating peritonitis (SEP), is an uncommon condition typically presenting with features of bowel obstruction. In this condition, the bowel is encapsulated or 'wrapped' with a thick fibrotic membrane like a cocoon ${ }^{[1,2]}$. The condition was first described in 1907 as peritonitis chronica fibrosa incapsulata. In 1978 it was further described by Foo et al. who coined the term 'abdominal cocoon' $[1,3]$. The name 'encapsulating peritoneal sclerosis' probably best describes the morphological and histological changes in this disorder ${ }^{[4]}$. 
This condition is one of the $6 \%$ of rare causes of bowel obstruction. In most cases it is diagnosed either by exclusion or as an intraoperative finding ${ }^{[5]}$. The aetiology of this condition is unclear but histopathological examination shows features of chronic inflammation. It may be either primary (idiopathic) or secondary in association with another conditions, particularly peritoneal dialysis or infectious disease, for example, tuberculosis ${ }^{[4,6]}$.

\section{CASE DESCRIPTION}

A normally fit 41-year-old male patient presented to the accident and emergency department with a 7-day history of abdominal pain which had been dull in character but had progressively worsened over the 3 days prior to presentation. There was associated nausea, repeated vomiting and relative constipation. The patient was a light smoker, consuming 5-10 cigarettes/day, and his only regular medication was tramadol for chronic lower back pain. He had no history of previous surgery. When asked, he mentioned that he has been experiencing vague diffuse pain all over his abdomen with difficulty opening his bowels for nearly 4 months. Apart from a low-grade fever of $37.9^{\circ} \mathrm{C}$, he did not show any haemodynamic instability. When initially reviewed, he had a pulse of $89 \mathrm{bpm}$ and a blood pressure of $130 / 70 \mathrm{mmHg}$.

On examination, the left hypochondrial region was mildly tender. A hard mass was palpable in the epigastric and periumbilical regions. Digital rectal examination showed an empty rectum.

Laboratory tests only showed a white cell count of 17 . The rest of the blood tests and the arterial gases were unremarkable. An abdominal $\mathrm{x}$-ray showed small intestinal dilatation which was mostly central. Contrast CT of the abdomen and pelvis was then ordered and was suggestive of small bowel obstruction involving most of the small bowel, with possible bowel wall thickening. There was no apparent transition point but mechanical obstruction could not be excluded (Figs. 1 and 2).

The decision was made to operate. The calculated P-Possum score showed potential morbidity of $21 \%$. At laparotomy, a tough whitish fibrous membrane was found encasing the entire length of the small bowel. Careful and slow excision of this membrane and adhesiolysis was undertaken (Fig. 3). A small perforation in an ileal loop around $50 \mathrm{~cm}$ from the caecum with multiple surrounding blackish spots was found (Fig. 4). We performed a limited resection of $10 \mathrm{~cm}$ of ileum with primary hand-sewn anastomosis using interrupted PDS 3-0 sutures. After the operation, the patient remained vitally stable and his pain significantly improved. He passed flatus on the 2 nd postoperative day and was allowed free oral fluids. His diet was gradually built up until he was discharged on the 5th postoperative day.

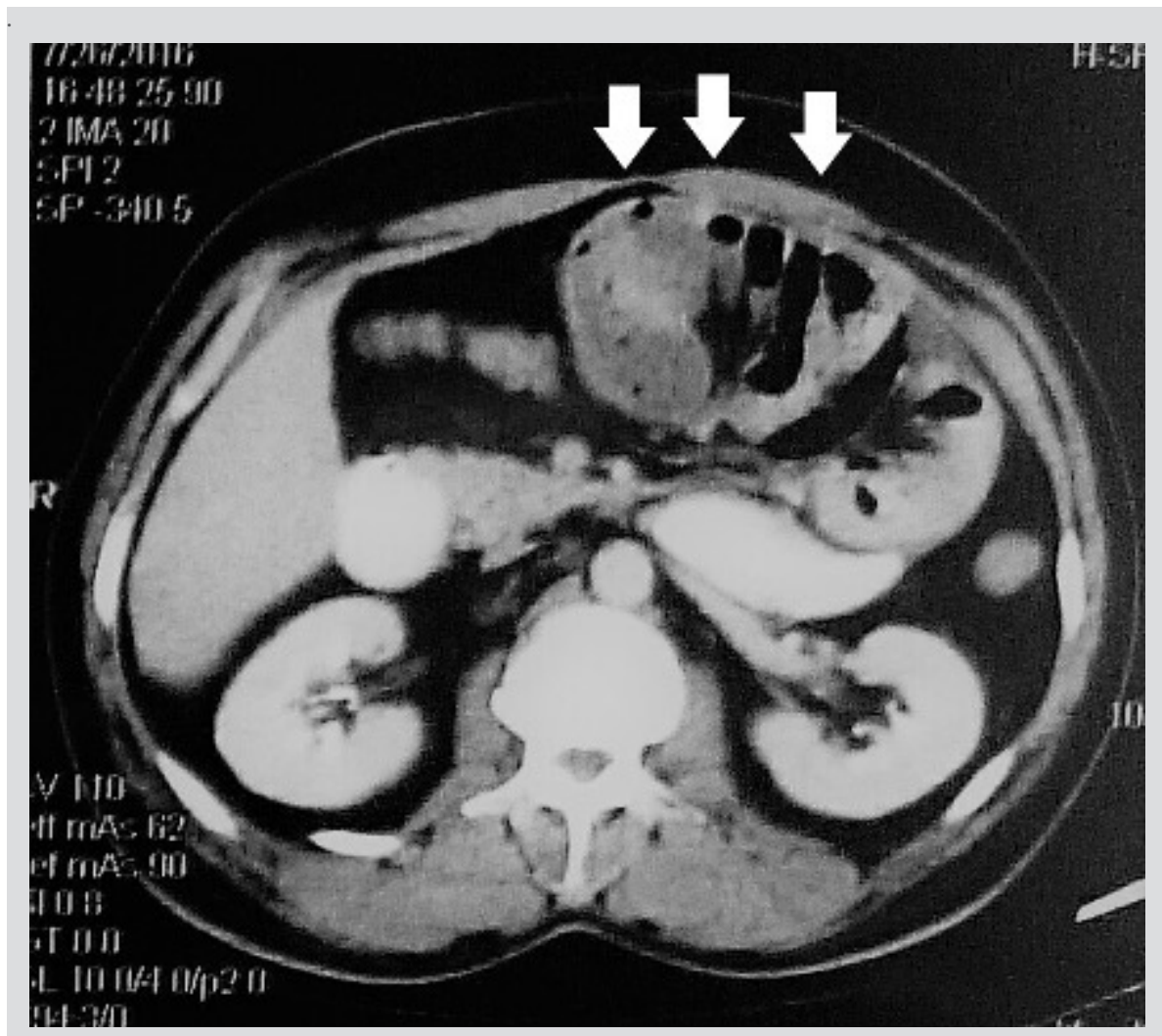

Figure 1. Contrast-enhanced CT of the upper abdomen showing a cluster of dilated small bowel loops in the centre giving a cauliflower appearance (arrows) 


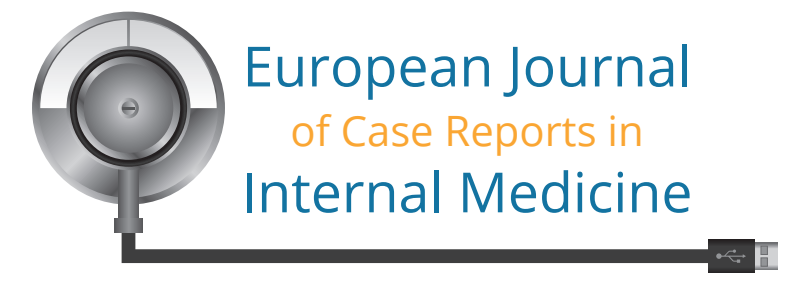

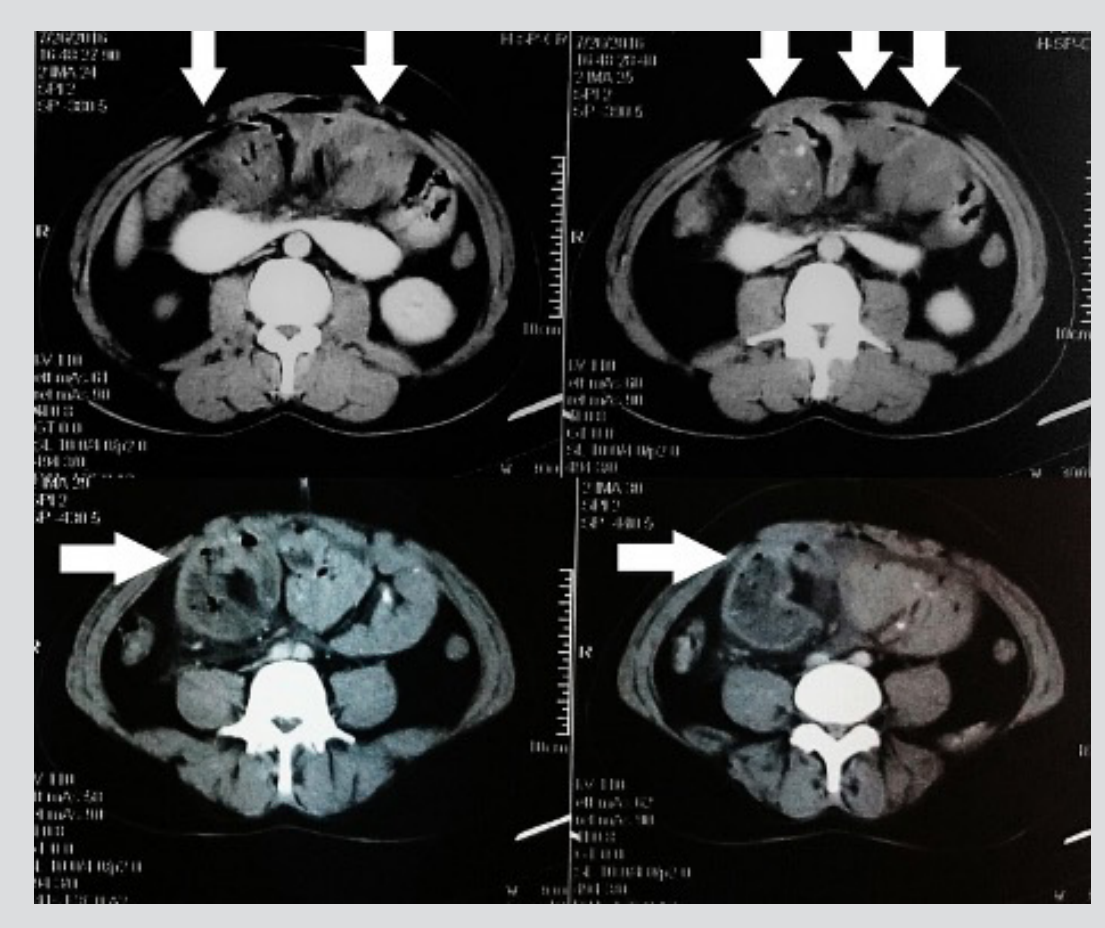
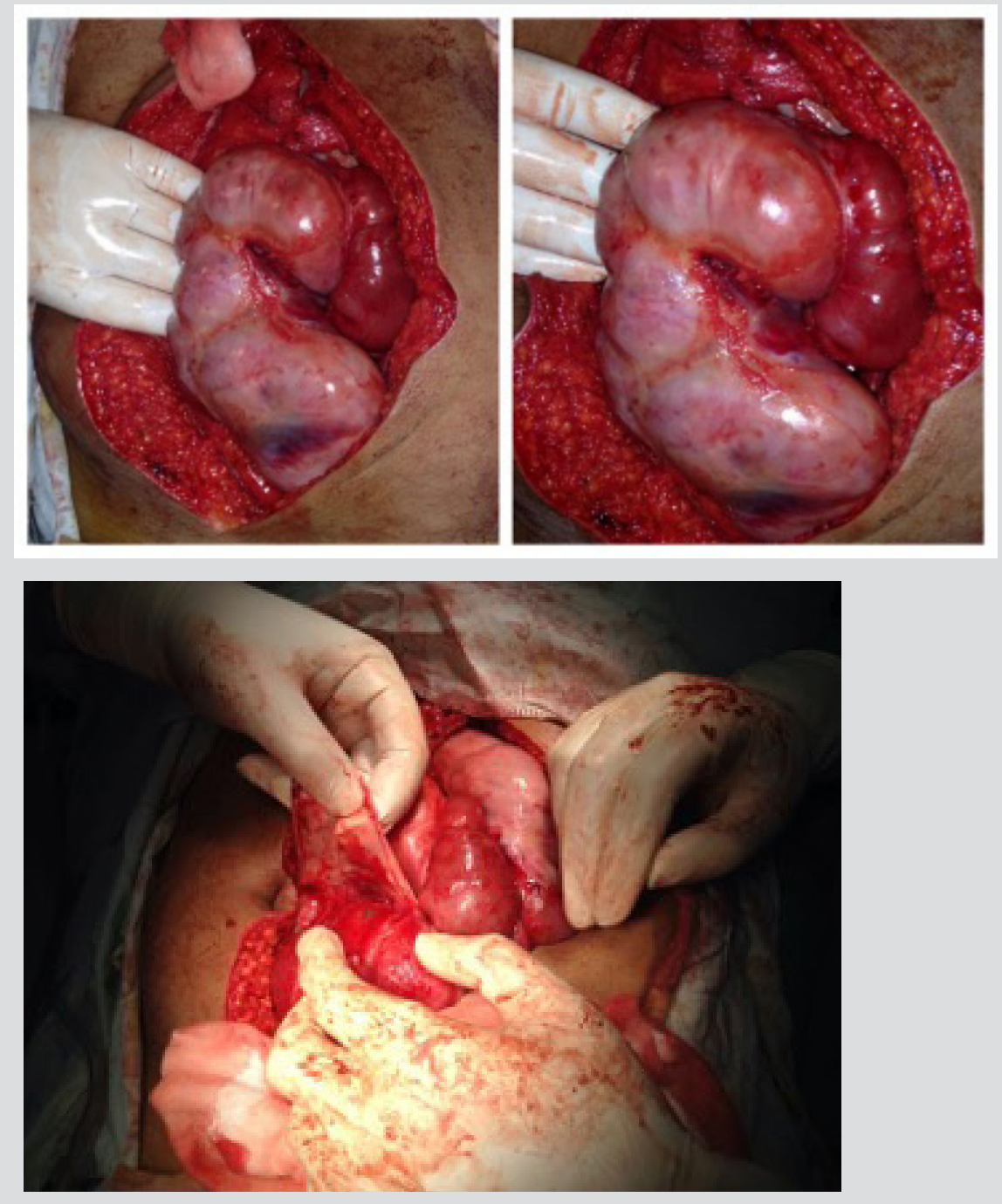

Figure 2. Contrast-enhanced CT of the abdomen showing the small bowel in the centre of the abdomen within a thick fibrous membrane (arrows)

Figure 3. Tough whitish fibrous membrane encasing the entire small bowel 


\section{DISCUSSION}

History and Terminology

EPS is a rare, potentially life-threatening condition in which the bowel is encased with a thick fibrous or fibrocartilaginous membrane like a cocoon. It can be either primary (idiopathic) or secondary (acquired). There has been growing concern relating a lot of secondary cases to prolonged peritoneal dialysis ${ }^{[4]}$. When first reported, it was suggested that EPS mostly affects young females. However, later it was found out that it may be discovered later in life and in males; only a few cases have been reported in the geriatric population [7] .

Other factors that could explain the disease must be absent in order for primary EPS to be accurately identified [8]. Initially it was though that early menstruation and primary peritonitis caused by retrograde menstruation might explain primary EPS, and hence it was thought to be mostly a disease of young females ${ }^{[2]}$. Several causes have been associated with the now more common entity of secondary EPS, although the exact mechanism remains unclear. These factors include prolonged peritoneal dialysis (the most common factor), peritoneal irritation due to surgical intervention or abdominal trauma, ventriculo-peritoneal shunts, infections (particularly peritoneal tuberculosis), prolonged practolol or propranolol (beta-blockers) therapy, intraperitoneal drug administration (e.g., chemotherapy or povidone), endometriosis, liver cirrhosis and neoplasms with peritoneal seeding (e.g., ovarian tumours), and connective tissue diseases (e.g., sarcoidosis and SLE) [2, 5]. Our patient had no medical history suggestive of any condition associated with secondary EPS, which leads us to believe that it might have been a case of primary EPS.

EPS can be categorized into three types according to the extent of the encasing membrane: type 1, the intestine is partially encapsulated by the membrane; type 2 , the entire intestine is encapsulated by the membrane; and type 3 , the entire intestine and other intraperitoneal organs such as the stomach, liver, appendix, caecum, colon and ovaries, are encapsulated by the membrane ${ }^{[8]}$.

EPS should generally be differentiated from peritoneal encapsulation, where the bowel lies behind a secondary but normal peritoneal layer which is attached to the ascending, descending and transverse colon. It is thought that this condition is embryogenic in origin and the sac is derived from the yolk sac. Unlike EPS, where most patients eventually require surgical management, most patients with peritoneal encapsulation have no or mild symptoms of abdominal pain and therefore it is mostly accidentally discovered on unrelated abdominal surgery or abdominal imaging $[4,6]$.

\section{Clinical Presentation}

Most patients with EPS have vague non-specific presentations which make the diagnosis challenging. Symptoms are usually comprised of vague abdominal pain and recurrent attacks of subacute intestinal obstruction which may have resolved on its own. Other presenting complaints include anorexia, weight loss, malnutrition and constipation ${ }^{[3,4]}$. More than $50 \%$ of patients present with a palpable non-tender abdominal mass ${ }^{[5]}$. Abdominal ascites might be detected in long-standing cases. The condition is usually misdiagnosed as adhesive intestinal obstruction, abdominal tumour or chronic appendicitis. Therefore, correct preoperative diagnosis is usually difficult and for most cases, the diagnosis of EPS is only established based on intraoperative finding ${ }^{[8]}$.

\section{Imaging}

Since most patients present with subacute intestinal obstruction, plain radiograms of the abdomen are usually normal in EPS, and bowel dilatation or air-fluid levels are usually absent. In secondary cases, especially those secondary to peritoneal dialysis, calcification might be seen. Contrast-enhanced computed tomography (CECT) has become the mainstay for diagnosis of EPS. In EPS, the peritoneum is thickened, with marked continuous enhancement. Assessment of peritoneal thickening is usually subjective, and no optimum threshold has been described, although a thickness greater than $2 \mathrm{~mm}$ appears to be a reasonable cut-off value. Usually, no obvious transition point is seen. CECT can also help exclude other conditions that might have similar presentations like internal hernias or visceral malignancy ${ }^{[4]}$. Several findings can be suggestive of EPS, such as clumping of bowel loops (small bowel loops with or without large bowel loops or other adjacent viscera), the cauliflower sign (defined as a broad 'head' formed by the bowel loops with a narrow mesenteric 'apex') and the bottle gourd sign (dilatation of the second and third parts of the duodenum with encasement of distal duodenum and jejunal loops) ${ }^{[9]}$.

\section{Management}

Surgery has been the traditional method to manage most cases, with laparotomy being the preferred approach. The role of laparoscopy is mostly limited to diagnostic purposes in doubtful cases, but in theory, it can be used for treatment only based on the surgeon's confidence and experience ${ }^{[5]}$. The typical intraoperative finding is encasement of the bowel (in its entirety or just partially) by a thick cocoon of fibrocartilaginous tissue. A possible association is hypoplasia or aplasia of the omentum. Surgery involves careful dissection and excision of the membrane combined with adhesiolysis ${ }^{[3,6]}$. It is believed that the risk of recurrence is low if the membrane is completely excised. Membrane dissection can be tedious and challenging and so extra attention should be given to avoid serosal tears or iatrogenic bowel injury, 
which unfortunately in some cases is inevitable. Bowel resection is reserved only for cases with non-viable or necrotic bowel ${ }^{[8]}$.

There is a role for medical and conservative management, particularly in cases with secondary EPS, but only if a preoperative diagnosis has been established and strong features of bowel obstruction or ischaemia are absent. Treatment should be tailored to each individual. For example, for cases associated with peritoneal dialysis, renal replacement can be exchanged with haemodialysis combined with total parenteral nutrition and the use of immunosuppressant or immunomodulatory drugs ${ }^{[4]}$. Drug treatments include colchicine, steroids, immunosuppressants (e.g., azathioprine) and tamoxifen ${ }^{[2]}$. In patients with tuberculous EPS, anti-tuberculous treatment should be started together with nasogastric decompression of the bowel, IV fluids and nutritional support. Failure of conservative/medical treatment is an indication for surgery ${ }^{[10]}$.

\section{CONCLUSION}

EPS remains an enigmatic condition and a rare cause of bowel obstruction. Advances in CT have made its diagnosis possible in some cases before a decision to intervene surgically is made. While surgery remains the usual management approach in most cases, conservative and medical treatment should be considered in mild cases and particularly in secondary peritoneal cases. Laparoscopy can be beneficial to establish a diagnosis in doubtful cases, and in expert hands can be a treatment option.

\section{REFERENCES}

1. Solak A, Solak I. Abdominal cocoon syndrome: preoperative diagnostic criteria, good clinical outcome with medical treatment and review of the literature. Turk J Gastroenterol 2012;23(6):776-779.

2. Habeeb MA, Vishwakarma SK, Abdul Wajid M, Bardia A, Khan AA, Bheerappa N. Abdominal cocoon: an enigma. Trop Gastroenterol 2015;36(2):130-131.

3. Gurleyik G, Emir S, Saglam A. The abdominal cocoon: a rare cause of intestinal obstruction. Acta Chir Belg 2010;110(3):396-398.

4. Singhal M, Krishna S, Lal A, Narayanasamy S, Bal A, Yadav TD, et al. Encapsulating peritoneal sclerosis: the abdominal cocoon. Radiographics 2019;39(1):62-77.

5. Mohakud S, Juneja A, Lal H. Abdominal cocoon: preoperative diagnosis on CT. BMJ Case Rep 2019;12(5):10-12.

6. Arif SH, Mohammed AA. Abdomen cocoon causing chronic abdominal pain and intestinal obstruction; a case series. Ann Med Surg 2019;48:7-10.

7. Deutsch GB, Shamonki J, Bilchik AJ. The cocoon abdomen. JAMA Surg 2014;149(11):1201-1202.

8. Xia J, Xie W, Chen L, Liu D. Abdominal cocoon with early postoperative small bowel obstruction: a case report and review of literature in China. Medicine (Baltimore) 2018;97(25):e11102.

9. Gorsi U, Gupta P, Mandavdhare HS, Singh H, Dutta U, Sharma V. The use of computed tomography in the diagnosis of abdominal cocoon. Clin Imaging 2018;50(2017):171-174.

10. Sharma V, Singh H, Mandavdhare HS. Tubercular abdominal cocoon: systematic review of an uncommon form of tuberculosis. Surg Infect (Larchmt) 2017 ; 18(6):736-741. 